\title{
Intuitionistic Fuzzy Set and Its Application in Corona Covid-19
}

\author{
Abdelmonem Mohamed Kozae ${ }^{1}$, Mohamed Shokry², Manar Omran 2, * \\ ${ }^{1}$ Department of Mathematics, Faculty of Science, Tanta University, Tanta, Egypt \\ ${ }^{2}$ Department of Physics and Engineering Mathematics, Tanta University, Tanta, Egypt
}

Email address:

akozae55@yahoo.com (A. M. Kozae),Omranmanar900@yahoo.com (M. Omran)

${ }^{*}$ Corresponding author

\section{To cite this article:}

Abdelmonem Mohamed Kozae, Mohamed Shokry, Manar Omran. Intuitionistic Fuzzy Set and Its Application In Corona Covid-19. Applied and Computational Mathematics. Vol. 9, No. 5, 2020, pp. 146-154. doi: 10.11648/j.acm.20200905.11

Received: July 14, 2020; Accepted: August 3, 2020; Published: September 3, 2020

\begin{abstract}
Intuitionistic Fuzzy set (IFS) theory plays an important role in real life and engineering problems. There are many model involving fuzzy matrices to deal with different complicated aspects. Intuitionistic fuzzy set (IFS) is useful in providing a flexible model for developing the uncertainty and vagueness involved in making decisions where the theories of uncertainty are very useful to treat with mathematics that needs to address. In other words, the application of intuitionistic fuzzy sets instead of fuzzy sets means the introduction of another degree of freedom into a set description. Intuitionistic fuzzy set (IFS) called the generalization of fuzzy sets was proposed in K. T. Atanassov. So, we can use it in decision making. We examined the definition of IFS and puts new definitions of IFS (Intuitionistic fuzzy set) in this paper and suggested its implementation in the Corona Covid-19. For several similar real-life cases the suggested approach can be applied.
\end{abstract}

Keywords: Fuzzy Sets, Intuitionistic Fuzzy Sets, Corona Covid-19, Corona Covid-19 Determination

\section{Introduction}

Fuzzy sets (FS) implemented in L. A. Zadeh [12] have shown important applications in a variety of fields of analysis. Fuzzy set concept is welcomed because it addresses ambiguity and vagueness that Cantorian set couldn't tackle. In fuzzy set theory, an element's membership in a Fuzzy set is a single value between zero and oneIn fact, however, the degree of non-membership of an entity in a fuzzy set cannot always be valid, as there may be some degree of hesitation. Therefore, K. T. Atanassov [9] proposed a generalization of fuzzy sets [7] as intuitionistic fuzzy sets (IFS) incorporating the degree of hesitation called the hesitation margin (And is defined as 1 minus the total number of degrees of membership and non-membership). In several areas of application, the notion of describing intuitive fuzzy set as a generalized fuzzy set is very interesting and useful. The knowledge and semantic representation of intuitionistic fuzzy set becomes more meaningful, resourceful and applicable because it includes the degree of belonging, the degree of non-belonging and the margin of hesitation K. T. Atanassov $[10,8]$. E. Szmidt et al. [4] showed that intuitionistic fuzzy sets are very useful in situations where a linguistic attribute represents a problem which it just seems to be too rough given in terms of membership features. Since of IFS 'versatility in managing ambiguity, they are a method for a more human-consistent reasoning under imperfectly specified facts and E. Szmidt et al. imprecise knowledge [5]. S. K. De et al. [14] presented an intuitive fuzzy approach in medical diagnosis using three measures such as: symptom determination, the formulation of medical information based on intuitive fuzzy relationships, and diagnosis determination based on the composition of intuitionistic fuzzy relationships. Intuitionistic fuzzy set is a method in modeling real-life problems such as sale analysis, new product promotion, financial services, negotiating phase, psychological investigations, etc. because there is a reasonable probability of a non-zero uncertainty component being present at each time an unknown object E. Szmidt et al. is evaluated. K. T. Atanassov [11] conducted thorough research on the theory and the applications of intuitionistic fuzzy sets. Many IFS applications are done using the approach of distance measures. In fuzzy mathematics, distance measurement between intuitionistic fuzzy sets is an important concept due 
to its broad applications in the real world, such as pattern recognition, machine learning, decision making and market forecasting. Several distance measurements between intuitionistic fuzzy sets have been proposed and studied in recent years by E. Szmidt et al. [3], W. Wang et al. [16] and used in medical diagnostics by E. Szmidt et al. [5]. We show a novel application of intuitionistic fuzzy set in a more challenging area of decision-making (i.e., choice of department [17]). The problem description uses the IFS concept, which allows two important facts to be rendered. To measure the distance between each student and each department, we use the Hamming distance method given in E. Szmidt et al. [6]. The smallest obtained value, points out a proper academic performance-based department determination.

\section{Concept of Intuitionistic Fuzzy Sets}

Definition 1 L. A. Zadeh [12]: Let A be non-empty. A whimsical set $\mathrm{A}$ from $\mathrm{X}$ is defined as $A=\left\{\left\langle\mathrm{x}, \mu_{A}(x)\right\rangle: \mathrm{x} \in \mathrm{X}\right\}$, where $\mu_{A}(x): \mathrm{X} \rightarrow[0,1]$ is the fuzzy set A membership function.

Fuzzy set is a collection of objects with membership gradation having membership degrees.

Definition 2 K. T. Atanassov [8]: Let X shall be a nonempty set. An intuitionistic fuzzy set $\mathrm{A}$ in $\mathrm{X}$ is an object that has the form $A=\left\{\left\langle\mathrm{x}, \mu_{A}(x), v_{A}(\mathrm{x})\right\rangle: \mathrm{x} \in \mathrm{X}\right\}$, where the functions $\mu_{A}(x), v_{A}(\mathrm{x}): \mathrm{X} \rightarrow[0,1]$ defines the degree of membership and the level of non-membership of the product, respectively $\mathrm{X} \in \mathrm{X}$ to the set $\mathrm{A}$, which is a subset of $\mathrm{X}$, and for every element $\mathrm{x} \in \mathrm{X}, 0 \leq \mu_{A}(x)+v_{A}(\mathrm{x}) \leq 1$
Furthermore, we have $\pi_{A}(x)=1-\mu_{A}(x)-v_{A}(\mathrm{x})$ called the intuitionistic fuzzy set index or hesitation margin of $\mathrm{x}$ in $\mathrm{A}$. $\pi_{A}(x)$ is the indeterminacy degree of $\mathrm{X} \in \mathrm{X}$ to the IFS $\mathrm{A}$ and $\pi_{A}(x) \in[0,1]$ i.e., $\pi_{A}(x): \mathrm{X} \rightarrow[0,1]$ and $0 \leq \pi_{A} \leq 1$ for every $x \in X . \pi_{A}(x)$ expresses a lack of information about whether or not $\mathrm{x}$ is part of IFS A.

Let A, for example, be an intuitionistic fuzzy set with $\mu_{A}(x)=0.5$ and $v_{A}(x)=0.3 \Rightarrow \pi_{A}(x)=1-(0.5+0.3)=$ 0.2 . The degree to which object $\mathrm{x}$ belongs to IFS $\mathrm{A}$ is 0.5 , the degree to which the object does not belong to IFS A is 0.3 and the degree of uncertainty is 0.2 .

\section{T-Norms and Archimedean T-Conorms in IFS}

In fuzzy set, we use t-norm to talk about the minimum between two memberships in intersection operation and use t-conorm to talk about the minimum between two memberships in union operation. So, we can use t-norm and $\mathrm{t}$-conorm in intuitionistic fuzzy set where it is useful to the intersection or the union operations that we need the minimum and maximum in the same operation in IFS $I=(x, \mu(x), v(x))$ where the intersection operation, the membership degree can use t-norm and the non-membership degree can use t-conorm and where the union operation, the membership degree can use t-conorm and the nonmembership degree can use t-norm. Consider that $\mathrm{i}$ is a $\mathrm{t}$ norm and $\mathrm{u}$ is a t-conorm. Considering that $x \in[0,1]$, we determine:

$$
\begin{gathered}
x_{i}^{(n)}=i(x, \ldots, x)=i\left(x_{i}^{(n-1)}, x\right) ; n \geq 2 \\
n-\text { times } \\
x_{u}^{(n)}=u(x, \ldots, x)=u\left(x_{u}^{(n-1)}, x\right) ; n \geq 2 \\
n \text {-times }
\end{gathered}
$$

Definition 3.1

Consider that $i$ and $u$ are a t-norm and a t-conorm from $[0,1]^{n}$ to $[0,1]$. Accordingly:

i. A t- norm $i$ (and a t-conorm $u$, respectively) is considered to be continuous if it continues as a function on the unit interval.

ii. A t-norm $i$ and a t-conorm $u$, respectively, of IFS $I=(x, \mu(x), v(x))$ if we talk about intersection operation: $\mu$ tends to minimum, $v$ tends to maximum is considered Archimedean if $\lim _{n \rightarrow \infty}\left(\mu_{i}(x)\right)^{n}=0$ and $\lim _{n \rightarrow \infty}\left(v_{u}(x)\right)^{n}=1$, respectively, for any.

iii. A t-norm $i$ and a t-c $x \in(0,1)$ onorm $u$, respectively, of IFS $I=(x, \mu(x), v(x))$ if we talk about union operation: $\mu$ tends to maximum, $v$ tends to minimum is considered as Archimedean if $\lim _{n \rightarrow \infty}\left(\mu_{i}(x)\right)^{n}=1$ and $\lim _{n \rightarrow \infty}\left(v_{u}(x)\right)^{n}=0$, respectively, for any $x \in(0,1)$.

Proposition 3.1

Consider that $\theta$ is a set of all IFS, $i$ and $u$ represent a t-norm and a t-conorm. Then: 
In intersection operation. If $i$ and $u$ are Archimedean, then

$i(\mu(x), \mu(x))<\mu(x)$ and $u(v(x), v(x))>v(x)$ for all $I=(x, \mu(x), v(x))$.

In union operation, if $\mathrm{i}$ and $\mathrm{u}$ are Archimedean, then $u(\mu(x), \mu(x))>\mu(x)$ and $i(v(x), v(x))<v(x)$ for all $I=(x, \mu(x), v(x))$.

Proof:

i. Consider the contrary, that's to say that $\mu(x)_{i}^{(2)}=\mathrm{i}(\mu(x), \mu(x)) \geq \mu(x)$ and $v(x)_{u}^{(2)}=u(v(x), v(x)) \leq v(x)$ for some $\quad x \in(0,1)$. Then, we have $\mu(x)_{i}^{(3)}=i(i(\mu(x), \mu(x)), \mu(x)) \geq i(\mu(x), \mu(x)) \geq \mu(x)$ and $v(x)_{u}^{(3)}=u(u(v(x), v(x)), v(x)) \leq u(v(x), v(x)) \leq v(x)$ from IFS properties and by inference we will also have

$$
\mu(x)_{i}^{(\mathrm{n})}=\mathrm{i}(\mu(x), \ldots, \mu(x)) \geq \mu(x) \text {. Then } \lim _{n \rightarrow \infty} \mu(x)_{i}^{(n)} \neq 0 \text { and }
$$$$
v(x)_{u}^{(\mathrm{n})}=u(v(x), \ldots, \quad v(x)) \leq v(x) . \text { Then } \lim _{n \rightarrow \infty} v(x)_{u}^{(n)} \neq 1 \text {, which is a contradiction. }
$$

ii. Consider the contrary, that's to say that $\mu(x)_{u}^{(2)}=u(\mu(x), \mu(x)) \leq \mu(x) \quad$ and

$$
v(x)_{i}^{(2)}=i(v(x), v(x)) \geq v(x) \text { for some } x \in(0,1) \text {. Then, we have }
$$

$\mu(x)_{u}^{(3)}=u(u(\mu(x), \mu(x)), \mu(x)) \leq \mathrm{u}(\mu(x), \mu(x)) \leq \mu(x) \quad$ and $\quad v(x)_{i}^{(3)}=i(i(v(x), v(x)), v(x)) \geq i(v(x), v(x)) \geq v(x)$ from the IFS properties and by inference we will also have:

$$
\mu(x)_{u}^{(\mathrm{n})}=u(\mu(x), \ldots, \mu(x)) \leq \mu(x) \quad \text {. Then } \quad \lim _{n \rightarrow \infty} \mu(x)_{u}^{(n)} \neq 1 \quad \text { and }
$$

$v(x)_{i}^{(\mathrm{n})}=i(v(x), \ldots, \quad v(x)) \geq v(x)$. Then, $\lim _{n \rightarrow \infty} v(x)_{i}^{(n)} \neq 0$, which a contradiction.

Proposition 3.2

In case of a continuous function where $i, u:[0,1]^{2} \rightarrow[0,1]$, there equivalent statements for any IFS as follows:

$i$ denotes a continuous Archimedean t-norm and $u$ is a continuous Archimedean t-conorm.

There are a continuous additive generator for any IFS $I=(x, \mu(x), v(x))$ if we talk about intersection operation $\mu$ tends to minimum, $v$ tends to maximum, that's to say that there exists a continuous strictly decreasing $i(\mu(x)):[0,1] \rightarrow[0, \infty], i(1)=0$ that is uniquely defined up to a multiplicative constant such that for all $x, y \in[0,1]$, we have:

$i(\mu(x), \mu(y))=i^{-1}(\min \{i(\mu(x))+i(\mu(y)), i(0)\})$ and strictly increasing $u(v(x)):[0,1] \rightarrow[0, \infty]$, $\mathrm{u}(1)=0$ that is uniquely defined up to a multiplicative constant such that for all $x, y \in[0,1]$, we will have:

$u(v(x), v(y))=u^{-1}(\max \{u(v(x))+u(v(y)), u(0)\})$

Proof:

Let $i(x, y)=i^{-1}(\min \{i(x)+i(y), i(0)\})$ And

$u(x, y)=u^{-1}(\max \{u(x)+u(y), u(0)\})$. We should prove that they are a continuous Archimedean t-norm and $\mathrm{t}-$ conorm.

$$
\begin{aligned}
I_{1}: i(\mu(x), 1) & =i^{-1}(\min \{i(\mu(x))+i(\mu(1)), i(0)\}) \\
& =i^{-1}(\min \{i(\mu(x))+0, i(0)\}) \\
& =i^{-1}(\min \{i(\mu(x)), i(0)\})=i^{-1}(i(\mu(x)))=\mu(x)
\end{aligned}
$$

And 


$$
\begin{aligned}
u(v(x), o) & =u^{-1}(\max \{u(v(x))+u(o), u(0)\}) \\
& =u^{-1}(\max \{u(v(x))+0, u(0)\}) \\
& =u^{-1}(\max \{u(v(x)), u(0)\})=u^{-1}(u(v(x)))=v(x)
\end{aligned}
$$

(Boundary).

$I_{2}$ : for proving that $i$ is increasing, let $\mu(x)<\mu(w)$ and $\mu(y)<\mu(z)$. Then, $i(\mu(x))>i(\mu(w))$ and $i(\mu(y))>i(\mu(z))$ and $i(\mu(x))+i(\mu(y))>i(\mu(w))+i(\mu(z))$. Since $i^{-1}$ is decreasing we obtain:

$$
\begin{aligned}
i(\mu(x), \mu(y)) & =i^{-1}(\min \{i(\mu(x))+i(\mu(y)), i(0)\}) \\
& \leq i^{-1}(\min \{i(\mu(w))+i(\mu(z)), i(0)\}) \leq i(\mu(w), \mu(z))
\end{aligned}
$$

And

For proving that $i$ is increasing, let $v(x)<v(w)$ and $v(y)<v(z)$. Then $u(v(x))>u(v(w))$ and $u(v(y))>u(v(z))$ and

$u(v(x))+u(v(y))>u(v(w))+u(v(z))$. Because $u^{-1}$ is increasing, we will have:

$$
\begin{aligned}
u(v(x), v(y)) & =u^{-1}(\max \{u(v(x))+u(v(y)), u(0)\}) \\
& \leq u^{-1}(\max \{u(v(w))+u(v(z)), u(0)\}) \leq u(v(w), v(z))
\end{aligned}
$$

(Monotonicity).

$I_{3}: i(\mu(x), \mu(y))=i(\mu(y), \mu(x))$ is obvious

And

$u(v(x), v(y))=u(v(y), v(x))$ is obvious (Commutative).

$I_{4}:$ We have:

$$
\begin{aligned}
& \left.i(i(\mu(x), \mu(y)), \mu(z))=i^{-1}\left(i^{-1}(\min \{i(\mu(x))+i(\mu(y)), i(0)\})\right), \mu(z)\right) \\
& \left.=i^{-1}\left(\min \left\{i\left(i^{-1}(\min \{i(\mu(x))+i(\mu(y)), i(0)\})\right)\right)+i(\mu(z)), i(0)\right\}\right)
\end{aligned}
$$

$\because i(\mu(x))+i(\mu(y))<i(0)$. Since $i^{-1}$ is strictly decreasing. We obtain:

$$
\begin{aligned}
i(i(\mu(\mathrm{x}), \mu(\mathrm{y})), \mu(\mathrm{z})) & =i^{-1}\left(\min \left\{i\left(i^{-1}(i(\mu(\mathrm{x}))+i(\mu(\mathrm{y})))\right)+i(\mu(\mathrm{z})), i(0)\right\}\right) \\
& =i^{-1}(\min \{(i(\mu(\mathrm{x}))+i(\mu(\mathrm{y}))+i(\mu(\mathrm{z})), i(0)\}), i(0)=1 \\
& =i^{-1}(i(\mu(\mathrm{x}))+i(\mu(\mathrm{y}))+i(\mu(\mathrm{z})))
\end{aligned}
$$

And

$$
\begin{aligned}
& \left.i(\mu(x), i(\mu(y), \mu(z)))=i^{-1}\left(\mu(x), i^{-1}(\min \{i(\mu(y))+i(\mu(z)), i(0)\})\right)\right) \\
& \left.=i^{-1}\left(\min \left\{i(\mu(x))+i\left(i^{-1}(\min \{i(\mu(y))+i(\mu(z)), i(0)\})\right)\right), i(0)\right\}\right)
\end{aligned}
$$

$\because i(\mu(y))+i(\mu(z))<i(0)$. Because $i^{-1}$ is decreasing strictly, we will have:

$$
\begin{aligned}
i(\mu(x), i(\mu(y), \mu(z))) & \left.=i^{-1}\left(\min \left\{\mathrm{i}(\mu(x))+i\left(i^{-1}(\mathrm{i}(\mu(y))+i(\mu(z)))\right)\right), i(0)\right\}\right) \\
& =i^{-1}(\min \{i(\mu(x))+i(\mu(y))+i(\mu(z)), i(0)\}), i(0)=1 \\
& =i^{-1}(i(\mu(x))+i(\mu(y))+i(\mu(z)))
\end{aligned}
$$

$\therefore i(i(\mu(x), \mu(y)), \mu(z))=i(\mu(x), i(\mu(y), \mu(z)))$

And we have: 
$\left.u(u(v(x), v(y)), v(z))=u^{-1}\left(u^{-1}(\min \{u(v(x))+u(v(y)), u(0)\})\right), v(z)\right)$

$\left.=u^{-1}\left(\min \left\{u\left(u^{-1}(\min \{u(v(x))+u(v(y)), u(0)\})\right)\right)+u(v(z)), u(0)\right\}\right)$

$\because u(v(x))+u(v(y))<u(0)$. Since $u^{-1}$ is strictly decreasing. We obtain:

$u(u(v(x), v(y)), v(z))=u^{-1}\left(\min \left\{u\left(u^{-1}(u(v(x))+u(v(y)))\right)+u(v(z)), u(0)\right\}\right)$

$$
\begin{aligned}
& =u^{-1}(\min \{(u(v(x))+u(v(y))+u(v(z)), u(0)\}), u(0)=1 \\
& =u^{-1}(u(v(x))+u(v(y))+u(v(z)))
\end{aligned}
$$

And

$$
\begin{aligned}
& \left.u(v(x), u(v(y), v(z)))=u^{-1}\left(v(x), u^{-1}(\min \{u(v(y))+u(v(z)), u(0)\})\right)\right) \\
& \left.=u^{-1}\left(\min \left\{u(v(x))+u\left(u^{-1}(\min \{u(v(y))+u(v(z)), u(0)\})\right)\right), u(0)\right\}\right)
\end{aligned}
$$

$\because u(v(y))+u(v(z))<u(0)$. Since $u^{-1}$ is strictly increasing. We obtain:

$$
\begin{aligned}
u(v(x), u(v(y), v(z))) & \left.=u^{-1}\left(\min \left\{u(v(x))+u\left(u^{-1}(u(v(y))+u(v(z)))\right)\right), u(0)\right\}\right) \\
& =u^{-1}(\min \{u(v(x))+u(v(y))+u(v(z)), u(0)\}), u(0)=1 \\
& =u^{-1}(u(v(x))+u(v(y))+u(v(z)))
\end{aligned}
$$

$\therefore u(u(v(x), v(y)), v(z))=u(v(x), u(v(y), v(z)))$ (Associative).

There exists a continuous additive generator for any IFS $I=(x, \mu(x), v(x))$ if we talk about intersection operation $\mu$ tends to maximum, $v$ tends to minimum i.e., there is strictly continuous decreasing $i(v(x)):[0,1] \rightarrow[0, \infty]$, $i(1)=0$ that is uniquely defined up to a multiplicative constant such that for all $x, y \in[0,1]$, we will obtain:

$i(v(x), v(y))=i^{-1}(\min \{i(v(x))+i(v(y)), i(0)\})$, and increasing strictly $u(\mu(x)):[0,1] \rightarrow[0, \infty]$, $u(1)=0$, that is uniquely defined up to a multiplicative constant such that for all $x, y \in[0,1]$, we will obtain:

$u(\mu(x), \mu(y))=u^{-1}(\max \{u(\mu(x))+u(\mu(y)), u(0)\})$

Proof:

Let $i(x, y)=i^{-1}(\min \{i(x)+i(y), i(0)\})$ And

$u(x, y)=u^{-1}(\max \{u(x)+u(y), u(0)\})$. We should prove that they are continuous Archimedean t-norm and tconorm.

$$
\begin{aligned}
I_{1}: i(v(x), 1) & =i^{-1}(\min \{i(v(x))+i(1), i(0)\}) \\
& =i^{-1}(\min \{i(v(x))+0, i(0)\}) \\
& =i^{-1}(\min \{i(v(x)), i(0)\})=i^{-1}(i(v(x)))=v(x)
\end{aligned}
$$

And

$$
\begin{aligned}
u(\mu(x), 0) & =u^{-1}(\max \{u(\mu(x))+u(0), u(0)\}) \\
& =u^{-1}(\max \{u(\mu(x))+0, u(0)\}) \\
& =u^{-1}(\max \{u(\mu(x)), u(0)\})=u^{-1}(u(\mu(x)))=\mu(x)
\end{aligned}
$$

(Boundary). 
$I_{2}$ : For proving that $i$ is increasing, let $v(x)<v(w)$ and $v(y)<v(z)$. Then $i(v(x))>i(v(w))$ and $\mathrm{i}(v(\mathrm{y}))>\mathrm{i}(v(\mathrm{z}))$ and

$i(\mathrm{v}(x))+i(\mathrm{v}(y))>i(\mathrm{v}(w))+i(\mathrm{v}(z))$. Because $i^{-1}$ is decreasing, we will have:

$$
\begin{aligned}
i(\mathrm{v}(x), \mathrm{v}(y)) & =i^{-1}(\min \{\mathrm{i}(\mathrm{v}(x))+\mathrm{i}(\mathrm{v}(y)), \mathrm{i}(0)\}) \\
& \leq i^{-1}(\min \{\mathrm{i}(\mathrm{v}(w))+\mathrm{i}(\mathrm{v}(z)), \mathrm{i}(0)\}) \leq i(\mathrm{v}(w), \mathrm{v}(z))
\end{aligned}
$$

And

For Proving that $i$ is increasing, let $\mu(x)<\mu(w)$ and $\mu(y)<\mu(z)$. Then $u(\mu(x))>u(\mu(w))$ and $u(\mu(y))>u(\mu(z))$ and $u(\mu(x))+u(\mu(y))>u(\mu(w))+u(\mu(z))$. Because $u^{-1}$ is increasing, we will have:

$$
\begin{aligned}
u(\mu(x), \mu(y)) & =u^{-1}(\max \{u(\mu(x))+u(\mu(y)), u(0)\}) \\
& \leq u^{-1}(\max \{u(\mu(w))+u(\mu(z)), u(0)\}) \leq u(\mu(w), \mu(z))
\end{aligned}
$$

(Monotonicity).

$I_{3}: i(v(x), v(y))=i(v(y), v(x))$ is obvious

And

$u(\mu(x), \mu(y))=u(\mu(y), \mu(x))$ is obvious (Commutative).

$I_{4}:$ We have:

$$
\begin{aligned}
& \left.i(i(v(x), v(y)), v(z))=i^{-1}\left(i^{-1}(\min \{\mathrm{u}(\mathrm{v}(x))+\mathrm{u}(\mathrm{v}(y)), \mathrm{u}(0)\})\right), \mathrm{v}(z)\right) \\
& \left.=i^{-1}\left(\min \left\{i\left(i^{-1}(\min \{\mathrm{u}(\mathrm{v}(x))+\mathrm{u}(\mathrm{v}(y)), \mathrm{u}(0)\})\right)\right)+\mathrm{u}(\mathrm{v}(z)), \mathrm{u}(0)\right\}\right)
\end{aligned}
$$

$\because i(v(x))+i(v(y))<i(0)$. Since $i^{-1}$ is strictly decreasing. We obtain:

$$
\begin{aligned}
i(i(v(x), v(y)), v(z)) & =i^{-1}\left(\min \left\{i\left(i^{-1}(i(v(x))+i(v(y)))\right)+i(v(z)), i(0)\right\}\right) \\
& =i^{-1}(\min \{(i(v(x))+i(v(y))+i(v(z)), i(0)\}), i(0)=1 \\
& =i^{-1}(i(v(x))+i(v(y))+i(v(z)))
\end{aligned}
$$

And

$$
\begin{aligned}
& \left.i(v(x), i(v(y), v(z)))=i^{-1}\left(v(x), i^{-1}(\min \{i(v(y))+i(v(z)), i(0)\})\right)\right) \\
& \left.=i^{-1}\left(\min \left\{i(v(x))+i\left(i^{-1}(\min \{i(v(y))+i(v(z)), i(0)\})\right)\right), i(0)\right\}\right)
\end{aligned}
$$

$\because i(v(y))+i(v(z))<i(0)$. Since $i^{-1}$ is strictly increasing. We obtain:

$$
\begin{aligned}
i(v(x), i(v(y), v(z))) & \left.=i^{-1}\left(\min \left\{i(v(x))+i\left(i^{-1}(i(v(y))+i(v(z)))\right)\right), i(0)\right\}\right) \\
& =i^{-1}(\min \{i(v(x))+i(v(y))+i(v(z)), i(0)\}), i(0)=1 \\
& =i^{-1}(i(v(x))+i(v(y))+i(v(z)))
\end{aligned}
$$

$\therefore i(i(v(x), v(y)), v(z))=i(v(x), i(v(y), v(z)))$

And we have:

$$
\begin{aligned}
& \left.u(u(\mu(x), \mu(y)), \mu(z))=u^{-1}\left(u^{-1}(\min \{u(\mu(x))+u(\mu(y)), u(0)\})\right), \mu(z)\right) \\
& \left.=u^{-1}\left(\min \left\{u\left(u^{-1}(\min \{u(\mu(x))+u(\mu(y)), u(0)\})\right)\right)+u(\mu(z)), u(0)\right\}\right)
\end{aligned}
$$


$\because u(\mu(x))+u(\mu(y))<u(0)$. Since $u^{-1}$ is strictly decreasing. We obtain:

$$
\begin{aligned}
u(u(\mu(x), \mu(y)), \mu(z)) & =u^{-1}\left(\min \left\{u\left(u^{-1}(u(\mu(x))+u(\mu(y)))\right)+u(\mu(z)), u(0)\right\}\right) \\
& =u^{-1}(\min \{(u(\mu(x))+u(\mu(y))+u(\mu(z)), u(0)\}), u(0)=1 \\
& =u^{-1}(u(\mu(x))+u(\mu(y))+u(\mu(z)))
\end{aligned}
$$

And

$$
\begin{aligned}
& \left.u(\mu(x), u(\mu(y), \mu(z)))=u^{-1}\left(\mu(x), u^{-1}(\min \{u(\mu(y))+u(\mu(z)), u(0)\})\right)\right) \\
& \left.=u^{-1}\left(\min \left\{u(\mu(x))+u\left(u^{-1}(\min \{u(\mu(y))+u(\mu(z)), u(0)\})\right)\right), u(0)\right\}\right)
\end{aligned}
$$

$\because u(\mu(y))+u(\mu(z))<u(0)$. Since $u^{-1}$ is strictly decreasing. We obtain:

$$
\begin{aligned}
u(\mu(x), u(\mu(y), \mu(z))) & \left.=u^{-1}\left(\min \left\{u(\mu(x))+u\left(u^{-1}(u(\mu(y))+u(\mu(z)))\right)\right), u(0)\right\}\right) \\
& =u^{-1}(\min \{u(\mu(x))+u(\mu(y))+u(\mu(z)), u(0)\}), u(0)=1 \\
& =u^{-1}(u(\mu(x))+u(\mu(y))+u(\mu(z)))
\end{aligned}
$$

$\therefore u(u(\mu(x), \mu(y)), \mu(z))=u(\mu(x), u(\mu(y), \mu(z)))$ (Associative).

Definition 2 E. Szmidt [2]:

The normalized Hamming distance $d_{n-H}(A, B)$ between two IFS A and B is defined as:

$$
d_{n-H}(A, B)=\frac{1}{2 n} \sum_{i=1}^{n}\left|\mu_{A}\left(x_{i}\right)-\mu_{B}\left(x_{i}\right)\right|+\left|v_{A}\left(x_{i}\right)-v_{B}\left(x_{i}\right)\right|+\left|\pi_{A}\left(x_{i}\right)-\pi_{B}\left(x_{i}\right)\right|, X=\left\{x_{1}, x_{2}, \ldots, x_{n}\right\} \text { for } i=1,2, \ldots, n .
$$

The normalized Euclidean distance $d_{n-H}(A, B)$ between two IFS A and B is defined as:

$$
d_{n-H}(A, B)=\left(\frac{1}{2 n} \sum_{i=1}^{n}\left(\mu_{A}\left(x_{i}\right)-\mu_{B}\left(x_{i}\right)\right)^{2}+\left(v_{A}\left(x_{i}\right)-v_{B}\left(x_{i}\right)\right)^{2}+\left(\pi_{A}\left(x_{i}\right)-\pi_{B}\left(x_{i}\right)\right)^{2}\right)^{1 / 2}, X=\left\{x_{1}, x_{2}, \ldots, x_{n}\right\} \text { for } i=1,2, \ldots, n .
$$

\section{Application of Intuitionistic Fuzzy Sets in Corona Covid-19}

The decision making Issues, especially in the case of medical diagnosis. At every moment of evaluation of an unknown object, there is a fair chance of the existence of a non-null hesitation part. To be more accurateintuitionist fuzzy sets let us convey, for example, the fact that a patient's temperature increases, and other symptoms are not quite obvious. In this paper in the presence of incomplete evidence and imprecise information we will present intuitionist fuzzy sets as a method for reasoning.

We use intuitionistic fuzzy sets as tool where there is a database, i.e. a description of a set of symptoms $\mathrm{S}$, and a set of diagnoses $\mathrm{D}$, an example of medical diagnosis will be presented. We'll describe a patient's state of knowledge of his / her medical test results that if he / she is sick with corona covid-19 or not. The problem analysis uses the notion of an intuitionistic Fuzzy package. The proposed diagnostic approach includes intuitionistic fuzzy distances, as introduced by $\mathrm{E}$. Szmidt, [2]. It includes three steps:

1) Determination of symptoms as shown in Table 1:

That success is represented by 3 numbers i.e. membership, margin of non-membership and hesitation. The students have obtained the following marks after the different exams as shown in the table below.

Table 1. Determination of Symptoms.

\begin{tabular}{llllll}
\hline Q & Temperature & Headache & Lost smell sense & Cough & Almond pain \\
\hline & $(0.8,0.1,0.1)$ & $(0.1,0.6,0.3)$ & $(0.8,0.2,0.0)$ & $(0.6,0.1,0.3)$ & $(0.6,0.1,0.3)$ \\
Al Bob Joe Ted & $(0.0,0.8,0.2)$ & $(0.4,0.4,0.2)$ & $(0.1,0.6,0.3)$ & $(0.1,0.7,0.2)$ & $(0.8,0.1,0.1)$ \\
& $(0.8,0.1,0.1)$ & $(0.1,0.8,0.1)$ & $(0.6,0.0,0.4)$ & $(0.2,0.7,0.1)$ & $(0.5,0.0,0.5)$ \\
& $(0.6,0.1,0.3)$ & $(0.4,0.5,0.1)$ & $(0.4,0.3,0.3)$ & $(0.7,0.2,0.1)$ & $(0.4,0.3,0.3)$ \\
\hline
\end{tabular}


Let the set of diagnoses be $\mathrm{D}=\{$ Corona Covid-19 or not $\}$. The considered set of symptoms is $\mathrm{S}=$ \{temperature, headache, Lost smell sense, cough, Almond pain $\}$.

The data are given in Table 2: each symptom is described by three numbers: membership $\mu$, non-membership $v$, hesitation margin $\pi$. For example, for Corona Covid-19: the temperature is high ( $\mu=0.7, v=0.0, \pi=0.3$ ), whereas for no Corona Covid-19: temperature is low ( $\mu=0.1, \nu=0: 8, \pi$ $=0: 1$ ). In fact, data is exactly the same, but we want to emphasize that the values of all three parameters are important in our approach by specifically including the hesitation margin.

Table 2. Formulation of Medical.

\begin{tabular}{ll}
\hline $\mathbf{R}$ & Corona Covid-19 jhhjhh \\
\hline Temperature & $(0.7,0.0,0.3)$ \\
Headache & $(0.6,0.2,0.2)$ \\
Lost smell sense & $(0.9,0.0,0.1)$ \\
Cough & $(0.7,0.0,0.3)$ \\
Almond pain & $(0.8,0.1,0.1)$ \\
\hline
\end{tabular}

The considered set of patients is $\mathrm{P}=\{\mathrm{Al}, \mathrm{Bob}, \mathrm{Joe}, \mathrm{Ted}\}$. The symptoms characteristic for the patients are given in Table 2 as before, we need all three parameters $(\mu, \nu, \pi)$ describing each symptom but the data are the same as in Table 1.

Our task is to make a proper diagnosis for each patient $p_{i}, \mathrm{i}=1, \ldots, 4$. To fulfill the task we propose to calculate for each patient pi a distance of his symptoms in Table 1 from a set of symptoms $s_{j}, \mathrm{j}=1, \ldots, 5$ characteristic for each diagnosis $d_{k}, \mathrm{k}=1, \ldots, 5$ in Table 2 . The lowest obtained distance points out a proper diagnosis.

In E. Szmidt [2] we have proven that the only proper way to measure the most commonly used distances for intuitive fuzzy sets is to take all three parameters into account: membership function, non-membership function and hesitation margin. To be more precise, this is described in (1) as the normalized Hamming distances for all the i-th patient's symptoms from the $\mathrm{k}$-th diagnosis.

The distances (1) for each patient from the considered set of possible diagnose are given in Table 3 . The greater than 0.5 suffers from Corona Covid-19: Bob suffers from Corona Covid-19.

2) Determination of diagnosis as shown in Table 3 and Table 4:

Table 3. Diagnosis Knowledge 1.

\begin{tabular}{ll}
\hline & Corona Covid-19 \\
\hline $\mathrm{Al}$ & 0.24 \\
$\mathrm{Bob}$ & 0.50 \\
Joe & 0.44 \\
Ted & 0.30 \\
\hline
\end{tabular}

Table 4. Diagnosis Knowledge 2.

\begin{tabular}{ll}
\hline & Corona Covid-19 \\
\hline Al & 0.25 \\
Bob & 0.56 \\
Joe & 0.41 \\
Ted & 0.29 \\
\hline
\end{tabular}

We obtained the same results, i.e. the same diagnosis of quality for each patient when searching for the solution while applying the standardized Euclidean distance defined in (2).

The results are given in Table 4: The greater than 0.5 suffers from Corona Covid-19. As before, Bob suffers from Corona Covid-19.

\section{Conclusion}

The case study presented in this paper can be applied to a lot of applications in real life. For instance: Political or social event. So, we deduce that intuitionistic fuzzy sets possess a uncertainty about examined objects in databases that we can communicate about. The method used, which performs diagnosis based on the calculation of distances from a considered case to all considered diseases, takes into consideration values of all symptoms. As a result, our method requires the use of weights for all symptoms (some symptoms may be more relevant for some illnesses).

\section{References}

[1] A. M Kozae, H. M. Abodonia, Granular computing, biapproximation spaces, 2005 IEEE international conference on Granular computing, 1, 167-170.

[2] E. Szmidt, Distances and similarities in intuitionistic fuzzy sets, Springer (2014).

[3] E. Szmidt, J. Kacprzyk, Distances between intuitionistic fuzzy Sets, Fuzzy Sets and Systems 114 (3) (2000) 505-518.

[4] E. Szmidt, J. Kacprzyk, Intuitionistic fuzzy sets in some medical applications, Note on IFS 7 (4) (2001) 58-64.

[5] E. Szmidt, J. Kacprzyk, Medical diagnostic reasoning using a Similarity measure for intuitionistic fuzzy sets, Note on IFS 10 (4) (2004) 61-69.

[6] E. Szmidt, J. Kacprzyk, On measuring distances between intuitionistic fuzzy Sets, Notes on IFS 3 (4) (1997) 1-3.

[7] K. T. Atanassov, Intuitionistic fuzzy sets, Fuzzy Sets and Systems 20 (1986) 87-96.

[8] K. T. Atanassov, Intuitionistic fuzzy sets: theory and application, Springer (1999).

[9] K. T. Atanassov, Intuitionistic fuzzy sets, VII ITKR's Session, Sofia, 1983.

[10] K. T. Atanassov, New operations defined over intuitionistic fuzzy sets, Fuzzy Sets and Systems Vol. 61, 2 (1994) 137-142.

[11] K. T. Atanassov, On Intuitionistic fuzzy sets, Springer (2012).

[12] L. A. Zadeh, Fuzzy sets, Information and Control 8 (1965) 338-353.

[13] MEA El-Monsef, A M Kozae, Al El-Maghrabi, Some Semi topologicalapplications on rough set, J. Egypt Math. Soc 12, 45-53, 2004.

[14] S. K. De, R. Biswas, A. R. Roy, An application of intuitionistic fuzzy sets in medical diagnostic, Fuzzy sets and systems 117 (2) (2001) 209-213. 
[15] T. K. Shinoj, J. J. Sunil, Intuitionistic Fuzzy multisets and it application in medical diagnosis, International journal of mathematical and computational sciences 6 (2012) 34-38.

[16] W. Wang, X. Xin, Distance measure between intuitionistic fuzzy sets. Pattern Recognition Letters 26 (2005) 2063-2069.
[17] A. M. Kozae, Assem Elshenawy, Manar Omran. Intuitionistic Fuzzy Set and Its Application in Selecting Specialization: A Case Study for Engineering Students. International Journal of Mathematical Analysis and Applications. Vol. 2, No. 6, 2015, pp. $74-78$. 